\title{
Manipulating Nanoscale Contact Electrification by an Applied Electric Field
}

\author{
Yu Sheng Zhou, ${ }^{\dagger}$ Sihong Wang, ${ }^{\dagger}$ Ya Yang, ${ }^{\dagger}$ Guang Zhu, ${ }^{\dagger}$ Simiao Niu, ${ }^{\dagger}$ Zong-Hong Lin, ${ }^{\dagger}$ Ying Liu, ${ }^{\dagger}$ \\ and Zhong Lin Wang*,
}

${ }^{\dagger}$ School of Materials Science and Engineering, Georgia Institute of Technology, North Ave NW, Atlanta, Georgia 30332-0245, United States

${ }^{\ddagger}$ Beijing Institute of Nanoenergy and Nanosystems, Chinese Academy of Sciences, No. 30 Xueyuan Road, Beijing 100083, China

ABSTRACT: Contact electrification is about the charge transfer between the surfaces of two materials in a contactseparation process. This effect has been widely utilized in particle separation and energy harvesting, where the charge transfer is preferred to be maximized. However, this effect is always undesirable in some areas such as electronic circuit systems due to the damage from the accumulated electrostatic charges. Herein, we introduced an approach to purposely manipulate the contact electrification process both in polarity and magnitude of the charge transfer through an applied electric field between two materials. Theoretical modeling and
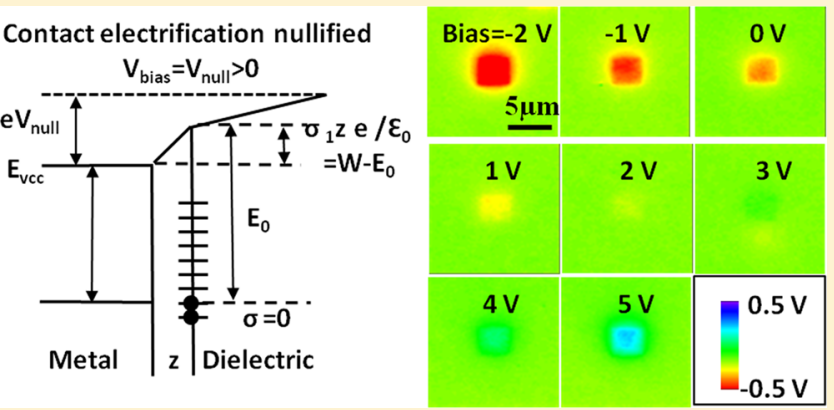
transfer between a Pt coated atomic force microscopy tip and Parylene film have been demonstrated. The modulation effect of the electric field on contact electrification is enhanced for a thinner dielectric layer. This work can potentially be utilized to enhance the output performance of energy harvesting devices or nullify contact electric charge transfer in applications where this effect is undesirable.

KEYWORDS: Contact electrification, atomic force microscopy, scanning Kelvin probe microscopy, nanogenerators

$\mathrm{C}$ ontact electrification is a universally existing phenomenon of the charge transfer between surfaces of two materials in a contact-separation process. ${ }^{1}$ It has been regarded as detrimental problems in a numbers of situations such as electronic circuits and systems, which thus attract extensive efforts to alleviate this effect. ${ }^{2}$ On the other hand, this effect has also been utilized for different purposes such as painting, ${ }^{3,4}$ particle separation, ${ }^{5}$ and mechanical energy harvesting, ${ }^{6,7}$ the last of which uses contact electric charges as electrostatic induction sources to generate electricity from mechanical energy in the form of impact, ${ }^{8,9}$ sliding, ${ }^{10-12}$ rotation, ${ }^{13}$ and so forth. For the sake of better performances in these applications, higher density of charges transferred in the contact electrification process is always favorable. ${ }^{14,15}$ Therefore, in all of the studies and applications related to the contact electrification, control of this effect is a critical issue. Previously, the modulation of contact-electric charge density was realized through intrinsic approaches such as material selection ${ }^{16,17}$ and surface functionalization ${ }^{18-20}$ that directly changes the structure of the two surfaces that are in contact. However, these methodologies are sometimes largely limited by the feasibility of material choices in some applications. So far, there have been few reports about the extrinsic method that can control charge transfer at insulator surfaces within preset materials. Using our recently developed atomic force microscopy (AFM) based in situ triboelectric characterization method, ${ }^{21}$ it is possible to investigate extrinsic approaches that can modulate the contact electrification process, which can be potentially utilized to enhance the output performance of energy harvesting devices or nullify the contact electric charge transfer in applications where this effect is undesirable.

In this paper, we investigate the effect of an extrinsically applied electric field on contact electrification between metals and dielectric films. A theoretical model was derived to elaborate the dependence of transferred charge density on different parameters such as externally applied bias and the thickness of dielectric layer at the equilibrium status. To verify the model, we employed the AFM based in situ characterization method. A Pt coated AFM tip was scanned over a Parylene surface with a bias, and the surface charge density is then characterized by the scanning Kelvin potential microscopy (SKPM). We found that both polarity and density of contact electric charge transfer can be modulated by the applied bias. At a certain bias, the contact electrification can be nullified so that no charge transfers between the two materials. The modulation effect is stronger on a thinner dielectric layer.

Results and Discussion. Figure 1a schematically displays the charge transfer between a metal and a dielectric layer in

Received: December 29, 2013

Revised: January 23, 2014 
(a)

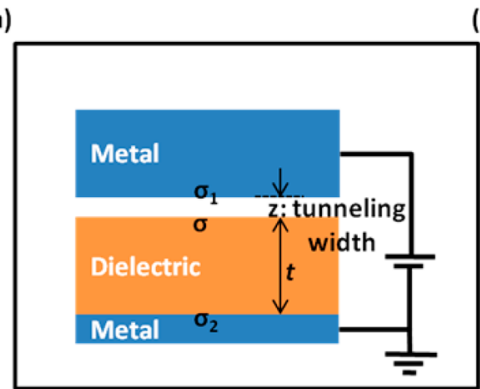

(d)

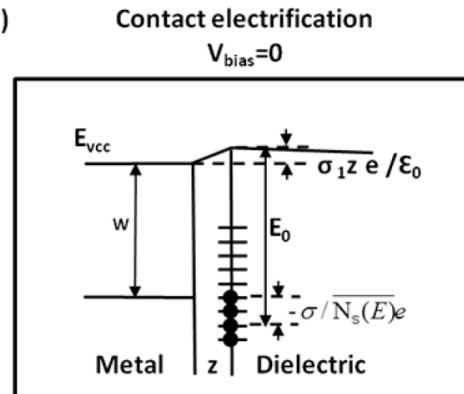

(b)

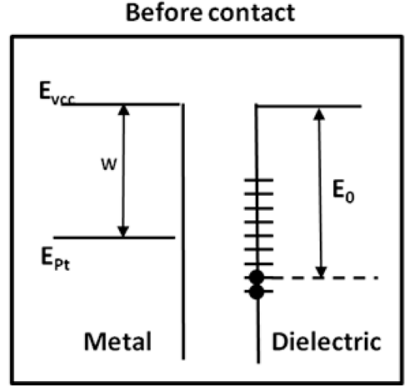

(e)

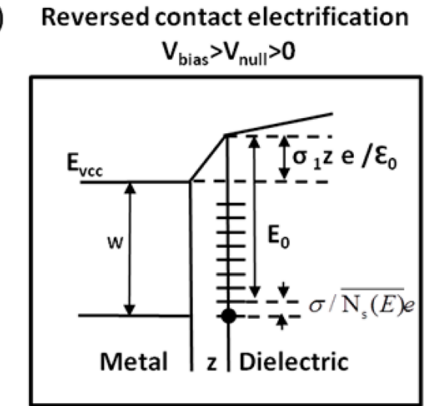

(c)

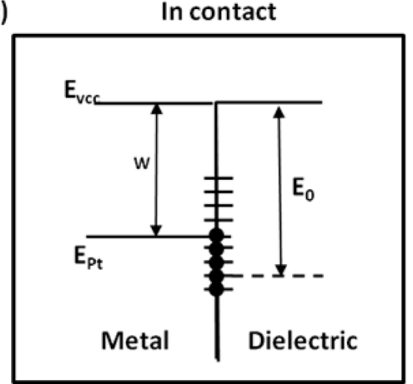

(f) Enhanced contact electrification

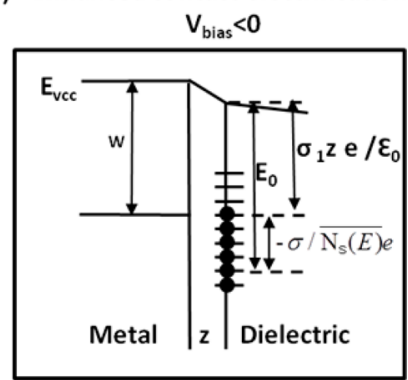

Figure 1. (a) Illustration of the contact electrification process with a tunneling width $z$, the charge transferred to the dielectric surface with a density of $\sigma$, induced charge density $\sigma_{1}$ and $\sigma_{2}$. (b-f) Energy band diagrams for the metal and dielectric materials in the situations of precontact (b), in contact with no bias (c), in separation equilibrium with no (d), positive (e), and negative (f) bias.
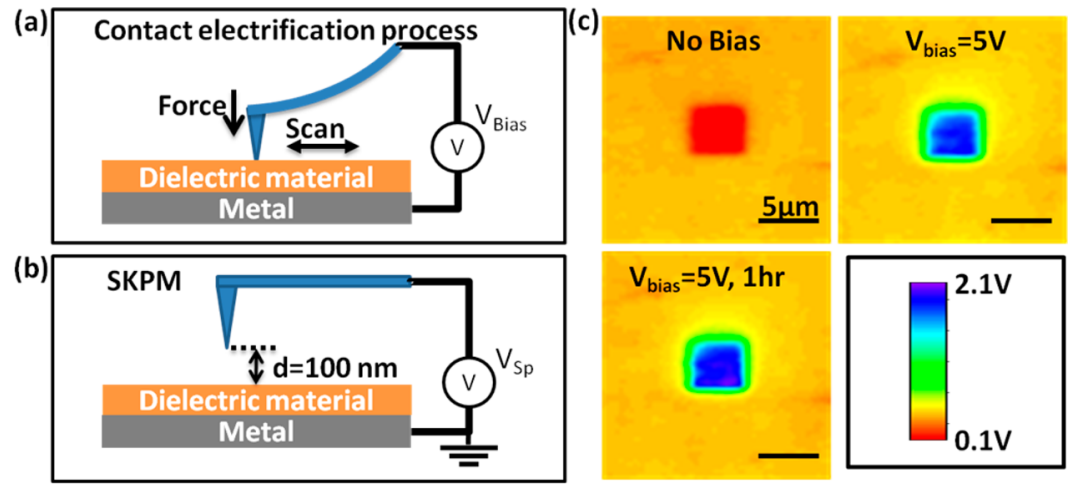

Figure 2. Schematic of experiment setup including two steps: (a) the first step is to use AFM tip with a bias between the tip and the substrate scan over the surface in contact mode, (b) the second step is to use SKPM to map the surface potential distribution. (c) Surface potential distribution of the rubbed and the surrounding area after regionally rubbed by AFM tip at no bias and $5 \mathrm{~V}$ bias immediately after rubbing and $1 \mathrm{~h}$ later, demonstrating that bias can reverse the triboelectric charge transfer, and the transferred charges have little leakage or diffusion.

contact electrification. When the top electrode contacts the dielectric layer, according to the electron transfer mechanism, ${ }^{22-24}$ electrons will transfer from one to the other due to the difference in the effective work functions of two materials. Subsequently, the transferred contact electric charges can also induce opposite charges in the two metal electrodes due to electrostatic induction. Although the equivalent band gap in dielectric materials are large (usually larger than $8 \mathrm{~V}$ ), it usually has surface states within the bandgap that can accommodate electrons. $^{22}$ Here we utilized the energy band diagrams to illustrate the case of a triboelectrically negative dielectric material (as compared to the metal). Accordingly, the highest filled surface energy states of the dielectric material is below the Fermi level of the metal, as illustrated in Figure 1b. When the two materials are in contact, electrons in the metal will flow from the top metal electrode onto the dielectric surface to fill up the surface states as high as the metal's Fermi level (Figure 1c). When two materials are separated from each other, an electric field is built up due to the transferred charge on the dielectric surface and image charges on the metal side, as depicted in Figure 1d. The strength of the electric field is proportional to the induced charge density $\sigma_{1}$ in the metal side. Due to this locally built-up electric potential, a portion of the charges on the dielectric surface can flow back to the metal. $^{1,22,25}$ The potential difference is determined by the separation distance and electric field strength. At the same time, the separation process also creates an energy barrier between two surfaces that hinders the back-flow of electrons. For simplicity, we assign a critical tunneling distance $z$ and assume that below $z$ the electrons can flow freely between two surfaces to maintain a constant Fermi level and above $z$ the barrier is large enough to prevent any tunneling. ${ }^{25}$ For a parallel-plates model, at the distance $z$, the dielectric surface charge $\sigma$ induces $\sigma_{1}$ on the top metal and $\sigma_{2}$ on the bottom metal, which should satisfy

$$
\sigma+\sigma_{1}+\sigma_{2}=0
$$


(a)
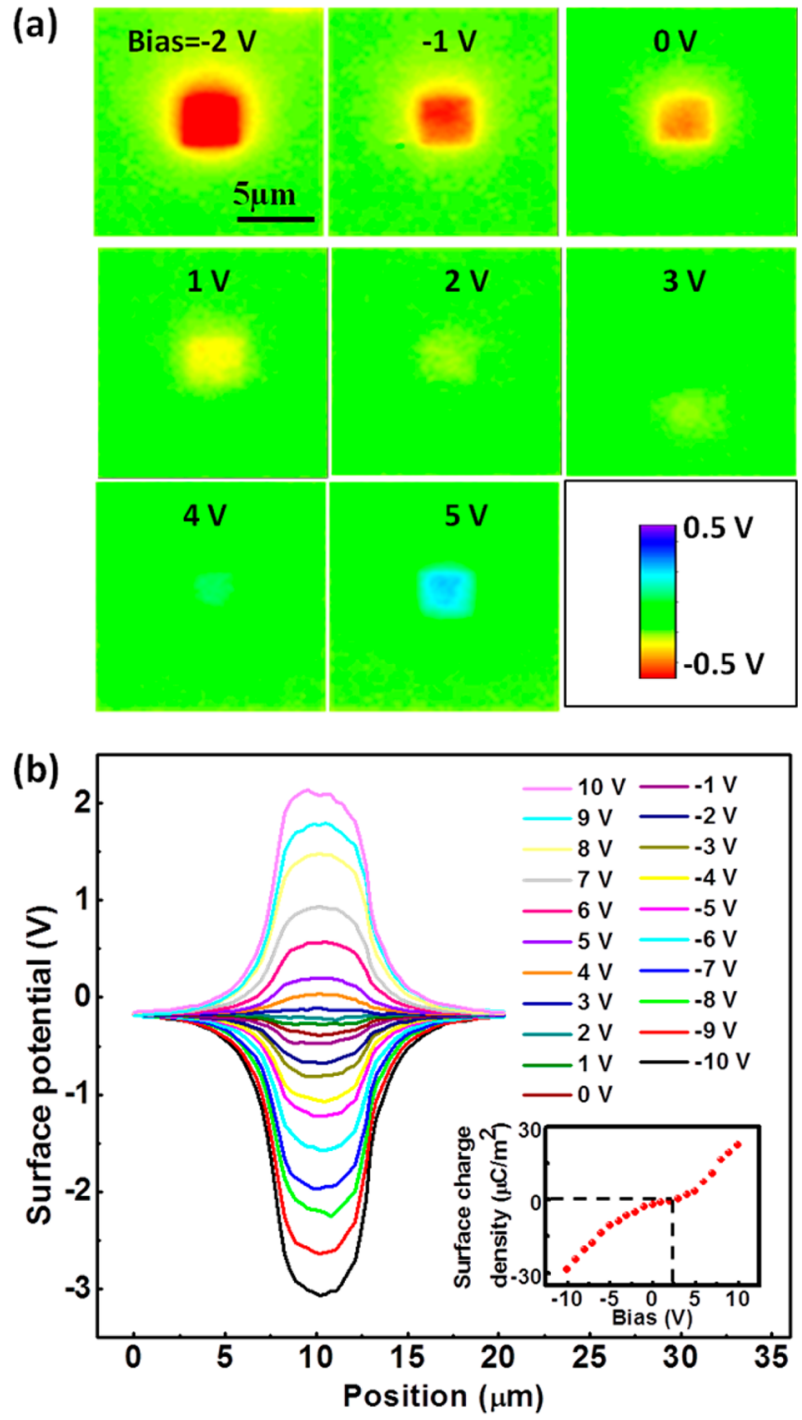

Figure 3. (a) Surface potential distributions of the Parylene film including the areas that were rubbed by Pt coated AFM tip at different bias from -2 to $5 \mathrm{~V}$. (b) Cross section profiles of the surface potential of the Parylene film rubbed with bias from -10 to $10 \mathrm{~V}$. The inset is the calculated surface charge density as a function of bias. With a bias of about $2.5 \mathrm{~V}$, the surface charge density is zero, indicating the contact electrification is completely canceled out by the applied bias.

Due to built-in electric field $\sigma_{1} / \varepsilon_{0}$, the vacuum energy level at the metal is reduced by $\Delta E_{\mathrm{vcc}}$ relative to the one at the dielectric side

$$
\Delta E_{\mathrm{vcc}}=\sigma_{1} z e / \varepsilon_{0}
$$

where $e$ is the elementary charge.

When the system is in equilibrium, the dielectric surface state will be filled up as high as the Fermi energy level in the metal. If we assume that the surface density of states is $N_{s}(E)$ and the range of filled surface states is $\Delta E_{\mathrm{s}}$, we have

$$
\sigma=-e \int_{E_{0}}^{E_{0}+\Delta E_{\mathrm{s}}} N_{\mathrm{s}}(E) \mathrm{d} E
$$

Here, we use the averaged surface density of states as defined below,

$$
\overline{N_{s}(E)}=\frac{\int_{E_{0}}^{E_{0}+\Delta E_{\mathrm{s}}} N_{\mathrm{s}}(E) \mathrm{d} E}{\Delta E_{\mathrm{s}}}
$$

Therefore, the range of filled surface states $\Delta E_{\mathrm{s}}$ can be described as

$$
\Delta E_{\mathrm{s}}=-\sigma / \overline{N_{\mathrm{s}}(E)} e
$$

Combining eq 2 and 5 , we have,

$$
E_{0}-W=\Delta E_{\mathrm{vcc}}+\Delta E_{\mathrm{s}}=\sigma_{1} z e / \varepsilon_{0}-\sigma / \overline{N_{\mathrm{s}}(E)} e
$$

An external potential applied between the top and the bottom (electrode underneath the dielectric film) metals will change the relative energy band height, which modulates the charge transfer accordingly. A positive bias applied to the top metal can lower its Fermi energy level, thus reducing the number of electrons transferred from the metal to the dielectric. At certain bias, the Fermi energy level will be as low as the highest filled surface states in the dielectric material. As a result, no charge will transfer between the metal and the dielectric surface, suggesting the contact electrification will be nullified. When the bias is more positive than the nullified bias, electrons will flow in a reversed way from the dielectric to the metal, leaving the dielectric surface positively charged, as illustrated in Figure 1e. On the contrary, a negative bias to the metal can raise its Fermi energy level, driving more electrons flow to the dielectric surface to fill up higher surface energy states, resulting in the dielectric surface to be more negatively charged (Figure 1f). Under the parallel-plate assumption, the bias $V$ between two metal electrodes can be written using the Poisson equation as:

$$
V=\frac{\sigma_{1}}{\varepsilon_{0}} z-\frac{\sigma_{2}}{\varepsilon \varepsilon_{0}} t
$$

Combining eqs 1,6 , and 7 , we can derive the surface charge density on the dielectric surface $\sigma$ as:

$$
\sigma=\frac{V+\left[\left(W-E_{0}\right) / e\right](1+t / \varepsilon z)}{t / \varepsilon \varepsilon_{0}+\left(1 / \overline{N_{s}(E)} e^{2}\right)(1+t / \varepsilon z)}
$$

This equation provides a guideline on how the external electric field quantitatively modulates the contact electrification.

To understand the proposed theoretical model, we present AFM based experimental results as follows. The design of our experiments is shown in Figure 2a and b. First, the AFM was operated in contact mode and the Pt coated tip scans over a certain area of a Parylene film (600 nm in thickness) under a bias between the substrate and the tip (Figure 2a). This process incurred contact electrification between the tip and the dielectric surface, and parameters such as bias, contact force, rubbing speed, and cycles can be accurately controlled in the AFM system. Subsequently, the surface potential of a larger area including the rubbed area were characterized in SKPM mode (Figure $2 \mathrm{~b}$ ) with the substrate grounded. The quantified surface potential difference between the rubbed and the intact areas can be used to calculate the surface charge density transferred by the contact electrification. Figure $2 \mathrm{c}$ shows the surface potential distribution of a $10 \times 10 \mu \mathrm{m}^{2}$ area including an area of $4 \times 4 \mu \mathrm{m}^{2}$ that was rubbed at 0 and $5 \mathrm{~V}$ biases before the measurement of surface potential, respectively. After zerobias contact electrification, the rubbed surface has a lower surface potential compared to the surrounding intact area, suggesting a negatively charged area. In contrast, after $5 \mathrm{~V}$ 

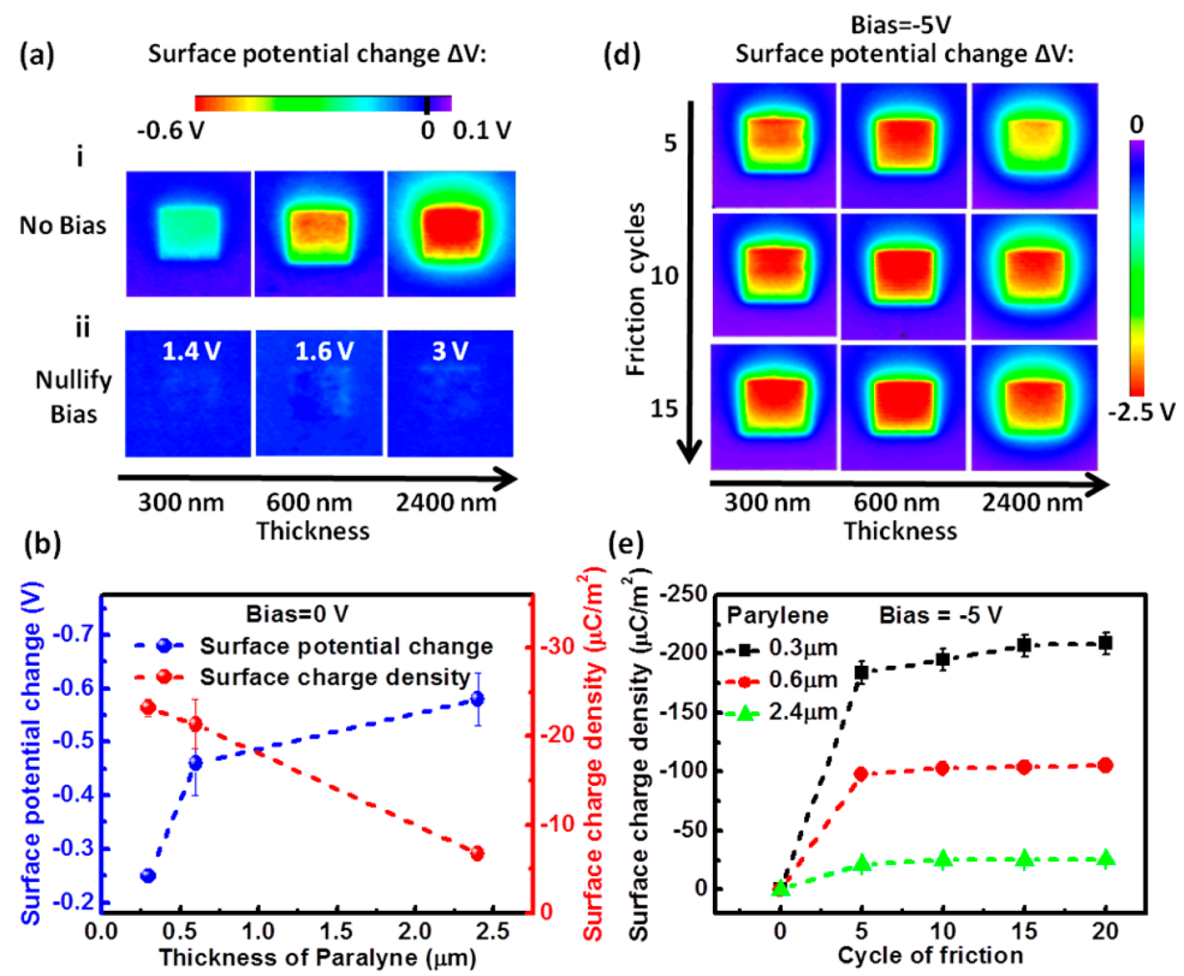

(e)
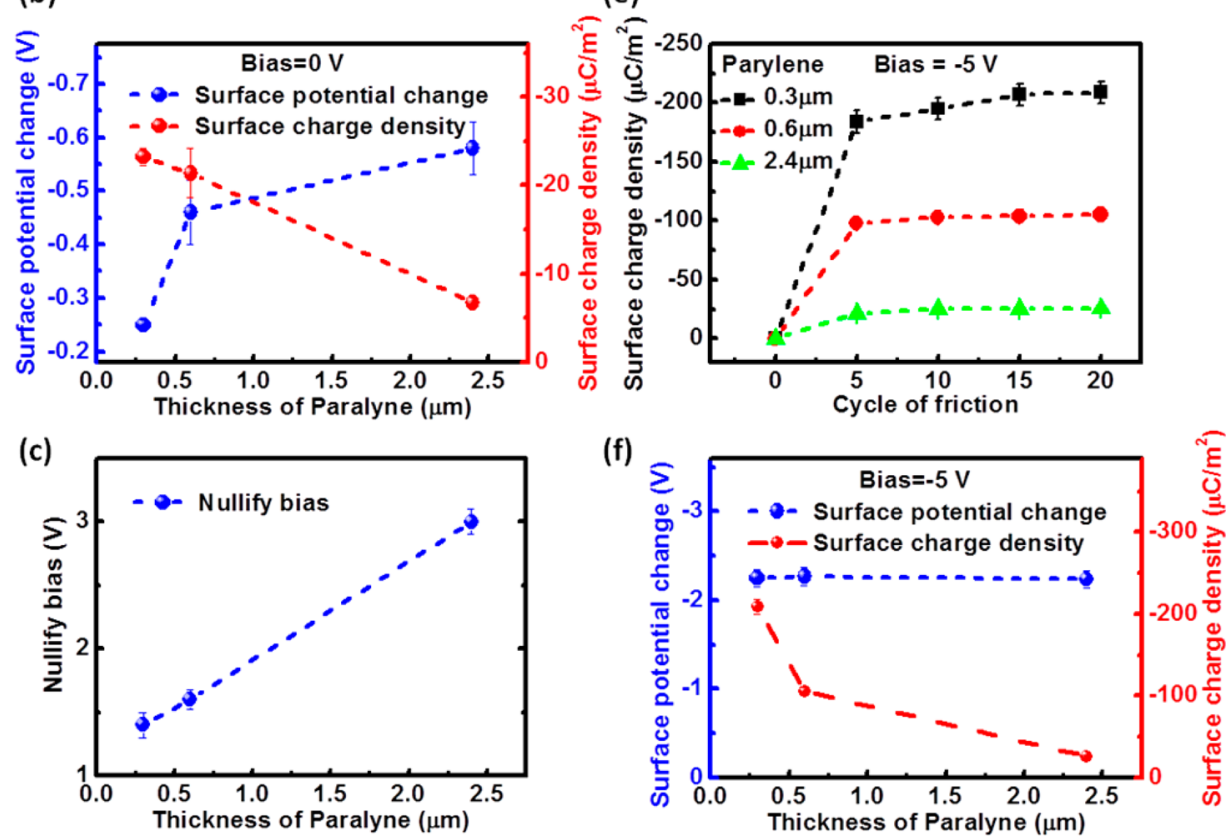

Figure 4. Thickness dependence of contact electrification and bias modulation. (a) Images of surface potential change $\Delta V$ of different thickness samples after they were rubbed at no bias (i) and nullify bias (ii), respectively. (b) The surface potential change and surface charge density as a function of the thickness of the Parylene layer after the sample was rubbed at no bias. (c) The nullify bias as a function of the thickness of the Parylene layer. (d) Images of surface potential change $\Delta V$ of different thickness samples after they were rubbed by different cycles. (e) Calculated surface charge density as a function of friction cycles. (f) The surface potential change and charge density after 20 cycles of friction as a function of thickness of Parylene.

biased contact electrification, the rubbed surface has a higher surface potential as compared to the surrounding intact area, indicating a positively charged area. The positive charges are locally preserved on the surface after $1 \mathrm{~h}$, suggesting that the reversely charged area is very stable. The experiment results are consistent with the proposed theoretical model in Figure 1e.

From eq 8 , the transferred charge density $\sigma$ should change with the externally applied bias $V$. To quantify the electric field influence, fresh areas of a Parylene film ( $2 \mu \mathrm{m}$ in thickness) were rubbed with different bias from -10 to $10 \mathrm{~V}$ and then measured in the SKPM mode. Figure 3a displays the surface potential distribution in the areas that were rubbed with biases from -2 to $5 \mathrm{~V}$ : a negative bias can enhance the negative charge density; a positive bias of $2 \mathrm{~V}$ almost nullified the charges; a bias of higher than $2 \mathrm{~V}$ brought positive charges to the rubbed surface. A cross section profile of the surface potential distribution of each experiment was plotted in Figure 3b. The surface potential of the rubbed area is adjusted monotonically by the applied bias, where the positive bias brings the surface potential to be more positive, and the negative bias exhibits the opposite effect. The corresponding surface charge density calculated from the surface potential profile is plotted as a function of the applied bias during the rubbing process in the inset of Figure $3 b .{ }^{21}$ It can be seen that the surface charge density increases with increasing the applied external bias with a nonlinear characteristic, which is associated with the different energy state densities in the middle of band gap and closer to the conduction/valence band. ${ }^{26}$

According to eq 8, the contact electrification and the modulation of applied bias should also be dependent on the thickness of the dielectric layer. We have conducted the experiments on three Parylene samples with different thicknesses of $300 \mathrm{~nm}, 600 \mathrm{~nm}$, and $2.4 \mu \mathrm{m}$. For the contact electrification without bias, the surface potential change increases with thickness, but the transferred surface charge density decreases with thickness, as shown in Figure 4 a(i) and b. Substituting $V=0$ into eq 8 , we have 


$$
\sigma=\frac{\left(W-E_{0}\right) / e}{1 /(\varepsilon / t+1 / z) \varepsilon_{0}+1 / \overline{N_{s}(E)} e^{2}}
$$

Equation 9 indicates that the conditions of a thinner dielectric layer and larger $\varepsilon / t$ can result in a higher surface charge density, which is consistent with the experimental results. In essence, this thickness dependence effect is because that the surface potential changed by contact charge decreases with thinner dielectric layer, and thus it takes more contact charges to make the upper filled energy level the same as Fermi level at the equilibrium.

The nullifying bias for different thickness also varies, as illustrated in Figure 4 a(ii) and c. For Parylene film with a thickness of $300 \mathrm{~nm}$, a bias of $1.4 \mathrm{~V}$ between the tip and the bottom electrode can leave the rubbed area with the same surface potential as the intact area, indicating that no charges were transferred during contact electrification process. The biases needed for nullification increases with increasing the thickness ( $1.4 \mathrm{~V}, 300 \mathrm{~nm} ; 1.6 \mathrm{~V}, 600 \mathrm{~nm} ; 3 \mathrm{~V}, 2.4 \mu \mathrm{m})$. From eq 8 , we can derive the nullify bias by substituting the surface charge density $\sigma$ with zero:

$$
V_{\text {null }}=\left(E_{0}-W\right) / e+\frac{t}{\varepsilon z}\left(E_{0}-W\right) / e
$$

The bias needed to nullify the contact electrification increases with the thickness of the dielectric film. It should be noticed that the relationship between the nullifying bias and the thickness of the dielectric layer may not be linear since the back tunneling width $z$ can also change with thickness. In atmospheric conditions, $z$ is more likely to be determined by the air breakdown electric field, ${ }^{27}$ which also varies with the dielectric thickness.

We also investigated the effect of dielectric thickness on the contact electric charge density in the bias-enhanced situation. In the experiment, for each sample, a $4 \times 4 \mu \mathrm{m}^{2}$ area was rubbed by the $-5 \mathrm{~V}$ biased tip for five cycles, and its surface potential was characterized in the SKPM mode. This process was repeated three times for the same area to obtain the information of the charging kinetics. As displayed in Figure 4e, the transferred surface charge density increases with friction cycles and saturates after 20 cycles. Figure $4 \mathrm{f}$ illustrates that the surface potential change is almost the same for different samples, while the surface charge density is larger for the thinner dielectric sample, indicating that the bias has a stronger effect on thinner samples for the surface charge transfer. This trend can also be implicated from eq 8 , where the slope of $V$ inversely correlates with the thickness $t$.

In conclusion, we report an approach to manipulate contact electrification process through an applied electric field. A theoretical model and following experiments between $\mathrm{Pt}$ and Parylene thin film presented in this study illustrates that the charges transferred from contact electrification can be modulated in both polarity and surface charge density by an external applied bias. A positive bias between Pt and Parylene can attenuate, nullify, or reverse the negative charges transferred from Pt onto Parylene surface, while a negative bias can be utilized to enhance the transferred negative charge density. Furthermore, this modulation effect is enhanced for thinner dielectric layers, and the nullifying bias increases with increasing dielectric thickness. Our study demonstrates an effective approach for controlling the contact electrification, with potential applications in charge-assisted separation, energy harvesting, as well as antistatic protection.
Materials and Methods. The Parylene samples used in the experiments were prepared by depositing Parylene C layer on the copper coated silicon wafer using SCS Labcoater PDS 2010. The thickness of the Parylene layer was controlled by the weight of the Parylene $C$ source and measured by SEM (SU8010 from Hitachi High-Tech) after deposition. During the experiment, a bias was applied between the AFM tip and the $\mathrm{Cu}$ layer by the AFM system (MFP-3D from Asylum Research). During contact mode, the deflection of the cantilever was set to be $0.4 \mathrm{~V}$, the sensitivity of the optical lever $126 \mathrm{~nm} / \mathrm{V}$, and the spring constant of the cantilever 0.98 $\mathrm{N} / \mathrm{m}$ and thus the normal force controlled at $49 \mathrm{nN}$.

\section{AUTHOR INFORMATION}

\section{Corresponding Author}

*E-mail: zlwang@gatech.edu.

\section{Notes}

The authors declare no competing financial interest.

\section{ACKNOWLEDGMENTS}

This research was supported by MANA, National Institute For Materials, Japan, a joint project with Sungkyunkwan University, Korea, and the "Thousands Talents" program for pioneer researchers and the innovation team, China, and Beijing City Committee of science and technology project (Z131100006013004, Z131100006013005).

\section{REFERENCES}

(1) Harper, W. R. Contact and frictional electrification; Laplacian Press: Morgan Hill, CA, 1998; p 369.

(2) Lorenz, R. D. Space Sci. Rev. 2008, 137, 287-294.

(3) Mazumder, M. K.; Banerjee, S.; Ware, R. E.; Mu, C.; Kaya, N.; Huang, C. C. IEEE Trans. Ind. Appl. 1994, 30, 365-369.

(4) Kleber, W.; Makin, B. Particul. Sci. Technol. 1998, 16, 43-53.

(5) Kwetkus, B. A. Particul. Sci. Technol. 1998, 16, 55-68.

(6) Fan, F. R.; Tian, Z. Q.; Wang, Z. L. Nano Energy 2012, 1, 328 334

(7) Fan, F. R.; Lin, L.; Zhu, G.; Wu, W. Z.; Zhang, R.; Wang, Z. L. Nano Lett. 2012, 12, 3109-3114.

(8) Wang, S. H.; Lin, L.; Wang, Z. L. Nano Lett. 2012, 12, 63396346.

(9) Zhu, G.; Pan, C. F.; Guo, W. X.; Chen, C. Y.; Zhou, Y. S.; Yu, R. M.; Wang, Z. L. Nano Lett. 2012, 12, 4960-4965.

(10) Zhu, G.; Chen, J.; Liu, Y.; Bai, P.; Zhou, Y. S.; Jing, Q. S.; Pan, C. F.; Wang, Z. L. Nano Lett. 2013, 13, 2282-2289.

(11) Yang, Y.; Zhang, H. L.; Chen, J.; Jing, Q. S.; Zhou, Y. S.; Wen, X. N.; Wang, Z. L. ACS Nano 2013, 7, 7342-7351.

(12) Wang, S. H.; Lin, L.; Xie, Y. N.; Jing, Q. S.; Niu, S. M.; Wang, Z. L. Nano Lett. 2013, 13, 2226-2233.

(13) Lin, L.; Wang, S. H.; Xie, Y. N.; Jing, Q. S.; Niu, S. M.; Hu, Y. F.; Wang, Z. L. Nano Lett. 2013, 13, 2916-2923.

(14) Niu, S. M.; Liu, Y.; Wang, S. H.; Lin, L.; Zhou, Y. S.; Hu, Y. F.; Wang, Z. L. Adv. Mater. 2013, 25, 6184-6193.

(15) Niu, S. M.; Wang, S. H.; Lin, L.; Liu, Y.; Zhou, Y. S.; Hu, Y. F.; Wang, Z. L. Energy Environ. Sci. 2013, 6, 3576-3583.

(16) Zhu, G.; Lin, Z. H.; Jing, Q. S.; Bai, P.; Pan, C. F.; Yang, Y.; Zhou, Y. S.; Wang, Z. L. Nano Lett. 2013, 13, 847-853.

(17) Diaz, A. F.; Felix-Navarro, R. M. J. Electrostat. 2004, 62, 277290.

(18) Lin, Z. H.; Xie, Y. N.; Yang, Y.; Wang, S. H.; Zhu, G.; Wang, Z. L. ACS Nano 2013, 7, 4554-4560.

(19) Horn, R. G.; Smith, D. T.; Grabbe, A. Nature 1993, 366, 442443.

(20) Trigwell, S.; Grable, N.; Yurteri, C. U.; Sharma, R.; Mazumder, M. K. IEEE Trans. Ind. Appl. 2003, 39, 79-86. 
(21) Zhou, Y. S.; Liu, Y.; Zhu, G.; Lin, Z. H.; Pan, C.; Jing, Q.; Wang, Z. L. Nano Lett. 2013, 13, 2771-2776.

(22) Lowell, J.; Roseinnes, A. C. Adv. Phys. 1980, 29, 947-1023.

(23) Wahlin, A.; Backstro, G. J. Appl. Phys. 1974, 45, 2058-2064.

(24) Arridge, R. G. C. Br. J. Appl. Phys. 1967, 18, 1311-1316.

(25) Lowell, J. J. Phys. D: Appl. Phys. 1979, 12, 1541-1554.

(26) Yeo, Y. C.; King, T. J.; Hu, C. M. J. Appl. Phys. 2002, 92, 72667271.

(27) Germer, L. H. J. Appl. Phys. 1959, 30, 46-51. 